PROCEEDINGS OF THE

AMERICAN MATHEMATICAL SOCIETY

Volume 138, Number 5, May 2010, Pages 1765-1773

S 0002-9939(09)10204-6

Article electronically published on December 16, 2009

\title{
PRODUCTS OF TOEPLITZ OPERATORS ON THE HARMONIC BERGMAN SPACE
}

\author{
XING-TANG DONG AND ZE-HUA ZHOU \\ (Communicated by Nigel J. Kalton)
}

\begin{abstract}
In this paper, we first discuss some basic results concerning Toeplitz operators with quasihomogeneous symbols (i.e., symbols being of the form $e^{i p \theta} \varphi$, where $\varphi$ is a radial function) on the harmonic Bergman space. Then we determine when the product of two Toeplitz operators with quasihomogeneous symbols is a Toeplitz operator.
\end{abstract}

\section{INTRODUCTION}

Let $d A$ denote the Lebesgue area measure on the unit disk $D$, normalized so that the measure of $D$ equals $1 . L^{2}(D, d A)$ is the Hilbert space of Lebesgue square integrable functions on $D$ with the inner product

$$
\langle f, g\rangle=\int_{D} f(z) \overline{g(z)} d A(z) .
$$

The harmonic Bergman space $L_{h}^{2}$ is the closed subspace of $L^{2}(D, d A)$ consisting of the harmonic functions on $D$. For $u \in L^{\infty}(D, d A)$, the Toeplitz operator $T_{u}$ with symbol $u$ is the operator on $L_{h}^{2}$ defined by

$$
T_{u} f=Q(u f)
$$

for $f \in L_{h}^{2}$, where $Q$ is the orthogonal projection of $L^{2}(D, d A)$ onto $L_{h}^{2}$. Each point evaluation is easily verified to be a bounded linear functional on $L_{h}^{2}$. Hence, for each $z \in D$, there exists a unique function $R_{z}$ (called the harmonic Bergman kernel) in $L_{h}^{2}$ that has the reproducing property

$$
f(z)=\left\langle f, R_{z}\right\rangle
$$

for every $f \in L_{h}^{2}$.

Given $z \in D$, let $K_{z}(w)=\frac{1}{(1-w \bar{z})^{2}}$ be the well-known reproducing kernel for the analytic Bergman space $L_{a}^{2}$ consisting of all $L^{2}$-analytic functions on $D$. The well-known Bergman projection $P$ is then the integral operator

$$
\operatorname{Pf}(z)=\int_{D} f(w) \overline{K_{z}(w)} d A(w)
$$

Received by the editors June 30, 2009, and, in revised form, September 7, 2009.

2010 Mathematics Subject Classification. Primary 47B35; Secondary 47 B38.

Key words and phrases. Toeplitz operators, harmonic Bergman space, quasihomogeneous symbols.

The second author was supported in part by the National Natural Science Foundation of China (Grant Nos. 10971153, 10671141).

(C)2009 American Mathematical Society Reverts to public domain 28 years from publication 
for $f \in L^{2}(D, d A)$. Since $L_{h}^{2}=L_{a}^{2}+\overline{L_{a}^{2}}$, it is easily checked that $R_{z}=K_{z}+\overline{K_{z}}-1$. Thus, $Q$ can be represented by

$$
Q f=P f+\overline{P \bar{f}}-P f(0) .
$$

Recall that a function $\varphi$ on $D$ is radial if $\varphi(z)$ depends only on $|z|$. Then for each radial function $\varphi$, we define the function $\widetilde{\varphi}$ on $[0,1)$ by $\widetilde{\varphi}(r)=\varphi\left(r e^{i \theta}\right)$, where $(r, \theta)$ are the polar coordinates of the complex variable $z$. It is obvious that $\widetilde{\varphi}$ is well defined. In the following, we shall often identify a bounded radial function $\varphi$ on $D$ with the corresponding function $\widetilde{\varphi}$ defined on $[0,1)$.

A bounded function $f$ is said to be quasihomogeneous of degree $p \in \mathbb{Z}$ if and only if

$$
f\left(r e^{i \theta}\right)=e^{i p \theta} \varphi(r)
$$

where $\varphi$ is a radial function (see [9]). In this case the associated Toeplitz operator $T_{f}$ is also called a quasihomogeneous Toeplitz operator of degree $p$.

In 1964, Brown and Halmos [2] proved that $T_{f} T_{g}=T_{h}$ on the Hardy space $H^{2}$ of the unit circle if and only if: either (I) $g$ is analytic, or (II) $f$ is conjugate analytic. They also showed that, in both cases $h=f g$. In the setting of an analytic Bergman space, conditions (I) and (II) are still sufficient, but they are no longer necessary. Ahern and Cučković [1] showed that a Brown-Halmos type result holds for Toeplitz operators with harmonic symbols on $L_{a}^{2}$. Later in [6], Louhichi, Strouse and Zakariasy gave necessary and sufficient conditions for the product of two quasihomogeneous Toeplitz operators to be a Toeplitz operator. Then Louhichi and Zakariasy [7] characterized commuting Toeplitz operators on $L_{a}^{2}$ with quasihomogeneous symbols. The main reason for them to study such a family of symbols is that any function $f$ in $L^{2}(D, d A)$ has the polar decomposition

$$
f\left(r e^{i \theta}\right)=\sum_{k \in \mathbb{Z}} e^{i k \theta} f_{k}(r)
$$

where $f_{k}$ are radial functions in $L^{2}([0,1], r d r)$. Recently, we studied some algebraic properties of quasihomogeneous Toeplitz operators on the analytic Bergman space of the unit ball in [5] and [10.

The theory of Toeplitz operators on $L_{h}^{2}$ is quite different from that on $L_{a}^{2}$. For example, Choe and Lee [3] showed that two analytic Toeplitz operators on $L_{h}^{2}$ commute only when their symbols and the constant function 1 are linearly dependent, but analytic Toeplitz operators always commute on $L_{a}^{2}$. In this paper, we shall consider when the product of two quasihomogeneous Toeplitz operators on $L_{h}^{2}$ is a Toeplitz operator.

We first give the following basic result concerning quasihomogeneous Toeplitz operators on $L_{h}^{2}$.

Theorem 1.1. Let $p \in \mathbb{Z}$ and let $f$ be a bounded function on $D$. Then the following assertions are equivalent:

(a) For each $k \in \mathbb{N}$, there exists $\lambda_{k} \in \mathbb{C}$ such that

$$
T_{f}\left(r^{k} e^{i k \theta}\right)=\lambda_{k} r^{|k+p|} e^{i(k+p) \theta} .
$$

(b) $f$ is a quasihomogeneous function of degree $p$.

Next, we show a necessary condition for the product of two quasihomogeneous Toeplitz operators on $L_{h}^{2}$ to be a Toeplitz operator. 
Theorem 1.2. Let $f_{1}$ and $f_{2}$ be two bounded quasihomogeneous functions on $D$ of degrees $k_{1}$ and $k_{2}$ respectively. If there exists a bounded function $h$ such that $T_{f_{1}} T_{f_{2}}=T_{h}$, then $h$ is a quasihomogeneous function of degree $k_{1}+k_{2}$.

Louhichi, Strouse and Zakariasy [6] gave lots of examples of radial functions $\psi_{1}$ and $\psi_{2}$ such that $T_{e^{i p \theta} \psi_{1}} T_{e^{-i s \theta} \psi_{2}}$ on $L_{a}^{2}$ is not a Toeplitz operator. Moreover, Theorem 6.7 of [6] illustrates the difficulty in characterizing more precisely those pairs of Toeplitz operators whose product is a Toeplitz operator. The problem of determining when the product of two quasihomogeneous Toeplitz operators on $L_{a}^{2}$ is a Toeplitz operators still remains open. The following theorem characterizes when the product of two Toeplitz operators on $L_{h}^{2}$ with certain quasihomogeneous symbols is a Toeplitz operator.

Theorem 1.3. Let $l_{1}, l_{2}>0$ and let $k_{1}, k_{2} \in \mathbb{Z}$. Then $T_{e^{i k_{1} \theta} r_{1} l_{1}} T_{e^{i k_{2} \theta} r_{2} l_{2}}$ is equal to a Toeplitz operator if and only if $k_{1}=k_{2}=0$. In this case:

$$
T_{r l_{1}} T_{r l_{2}}= \begin{cases}\frac{l_{1}}{l_{1}-l_{2}} T_{r l_{1}}-\frac{l_{2}}{l_{1}-l_{2}} T_{r l_{2}} & \text { if } l_{1} \neq l_{2} \\ T_{r l_{1}\left(1+l_{1} \log r\right)} & \text { if } l_{1}=l_{2} .\end{cases}
$$

\section{Some PRELIMINARY RESUlts}

One of the most useful tools in the following calculations will be the Mellin transform.

The Mellin transform $\hat{\varphi}$ of a function $\varphi \in L^{1}([0,1], r d r)$ is defined by the equation

$$
\hat{\varphi}(z)=\int_{0}^{1} \varphi(s) s^{z-1} d s .
$$

It is clear that $\hat{\varphi}$ is well defined on the right half-plane $\{z: \operatorname{Re} z \geq 2\}$ and analytic on $\{z: \operatorname{Re} z>2\}$. The use of the Mellin transform in the study of Toeplitz operators was introduced for the first time in [4. A direct calculation gives the following lemma, which we shall use often.

Lemma 2.1. Let $p \in \mathbb{Z}$ and let $\varphi$ be a bounded radial function. Then for each $k \in \mathbb{N}$,

$$
\begin{aligned}
& T_{e^{i p \theta} \varphi}\left(z^{k}\right)= \begin{cases}2(k+p+1) \widehat{\varphi}(2 k+p+2) z^{k+p} & \text { if } k \geq-p \\
2(-k-p+1) \widehat{\varphi}(-p+2) \bar{z}^{-k-p} & \text { if } k<-p,\end{cases} \\
& T_{e^{i p \theta} \varphi}\left(\bar{z}^{k}\right)= \begin{cases}2(k-p+1) \widehat{\varphi}(2 k-p+2) \bar{z}^{k-p} & \text { if } k \geq p \\
2(p-k+1) \widehat{\varphi}(p+2) z^{p-k} & \text { if } k<p .\end{cases}
\end{aligned}
$$

Proof. Since $\varphi$ is a bounded radial function, we see that for each $k \in \mathbb{N}$,

$$
\begin{aligned}
P\left(e^{i p \theta} \varphi w^{k}\right)(z) & =\int_{D} \varphi(r) e^{i p \theta} w^{k} \overline{K_{z}(w)} d A(w) \\
& =\sum_{j=0}^{\infty}(j+1) z^{j} \int_{D} \varphi(r) e^{i p \theta} w^{k} \bar{w}^{j} d A(w) \\
& = \begin{cases}2(k+p+1) \widehat{\varphi}(2 k+p+2) z^{k+p} & \text { if } k \geq-p \\
0 & \text { if } k<-p\end{cases}
\end{aligned}
$$


and

$$
\begin{aligned}
P\left(e^{i p \theta} \varphi \bar{w}^{k}\right)(z) & =\int_{D} \varphi(r) e^{i p \theta} \bar{w}^{k} \overline{K_{z}(w)} d A(w) \\
& =\sum_{j=0}^{\infty}(j+1) z^{j} \int_{D} \varphi(r) e^{i p \theta} \bar{w}^{k+j} d A(w) \\
& = \begin{cases}2(p-k+1) \widehat{\varphi}(p+2) z^{p-k} & \text { if } k \leq p \\
0 & \text { if } k>p .\end{cases}
\end{aligned}
$$

Note that $\bar{\varphi}$ is still radial and

$$
\overline{\widehat{\varphi}(n)}=\widehat{\bar{\varphi}}(n), \quad \forall n \in \mathbb{N} .
$$

Thus,

$$
\begin{aligned}
T_{e^{i p \theta} \varphi}\left(w^{k}\right)(z) & =P\left(e^{i p \theta} \varphi w^{k}\right)(z)+\overline{P\left(\bar{\varphi} e^{-i p \theta} \bar{w}^{k}\right)}(z)-P\left(e^{i p \theta} \varphi w^{k}\right)(0) \\
& = \begin{cases}2(k+p+1) \widehat{\varphi}(2 k+p+2) z^{k+p} & \text { if } k \geq-p \\
2(-k-p+1) \widehat{\varphi}(-p+2) \bar{z}^{-k-p} & \text { if } k<-p\end{cases}
\end{aligned}
$$

and

$$
\begin{aligned}
T_{e^{i p \theta} \varphi}\left(\bar{w}^{k}\right)(z) & =P\left(e^{i p \theta} \varphi \bar{w}^{k}\right)(z)+\overline{P\left(\bar{\varphi} e^{-i p \theta} w^{k}\right)}(z)-P\left(e^{i p \theta} \varphi \bar{w}^{k}\right)(0) \\
& = \begin{cases}2(k-p+1) \widehat{\varphi}(2 k-p+2) \bar{z}^{k-p} & \text { if } k \geq p \\
2(p-k+1) \widehat{\varphi}(p+2) z^{p-k} & \text { if } k<p .\end{cases}
\end{aligned}
$$

This completes the proof.

Remark 2.2. Let $p, s \in \mathbb{Z}$. Then Lemma 2.1 implies that the image of $r^{|s|} e^{i s \theta}$ by a quasihomogeneous Toeplitz operator of degree $p$ is $\lambda_{p, s} r^{|p+s|} e^{i(p+s) \theta}$ for some constant $\lambda_{p, s}$.

Lemma 2.3. Let $f$ be a bounded function on D. Then the following conditions are equivalent:

(a) For any $k \in \mathbb{N}$, there exists $\lambda_{k} \in \mathbb{C}$ such that $T_{f}\left(z^{k}\right)=\lambda_{k} z^{k}$.

(b) For any $k \in \mathbb{N}$, there exists $\lambda_{k} \in \mathbb{C}$ such that $T_{f}\left(\bar{z}^{k}\right)=\lambda_{k} \bar{z}^{k}$.

Proof. First suppose that $(a)$ holds, so $T_{f}\left(z^{k}\right)=\lambda_{k} z^{k}$ for each $k \in \mathbb{N}$. Then for any $m \in \mathbb{N}$, we have

$$
\begin{aligned}
\left\langle T_{f} \bar{z}^{k}, \bar{z}^{m}\right\rangle & =\left\langle f z^{m}, z^{k}\right\rangle=\left\langle\lambda_{m} z^{m}, z^{k}\right\rangle \\
& = \begin{cases}\lambda_{k}\left\langle z^{k}, z^{k}\right\rangle & \text { if } m=k \\
0 & \text { if } m \neq k,\end{cases}
\end{aligned}
$$

which implies that

$$
\left\langle T_{f} \bar{z}^{k}, \bar{z}^{m}\right\rangle=\left\langle\lambda_{k} \bar{z}^{k}, \bar{z}^{m}\right\rangle \text {. }
$$

Also, a similar calculation shows that

$$
\left\langle T_{f} \bar{z}^{k}, z^{m}\right\rangle=\left\langle\lambda_{k} \bar{z}^{k}, z^{m}\right\rangle .
$$

Since $\left\{\sqrt{k+1} z^{k}\right\}_{k=0}^{\infty} \cup\left\{\sqrt{k+1} \bar{z}^{k}\right\}_{k=1}^{\infty}$ is an orthonormal basis for the harmonic Bergman space, we have

$$
T_{f} \bar{z}^{k}=\lambda_{k} \bar{z}^{k}
$$

for any $k \in \mathbb{N}$.

By a similar argument, one can also show that (b) implies (a). This completes the proof. 
When considering the product of two Toeplitz operators we often use a wellknown fact about the Mellin convolution of their symbols. If $f$ and $g$ are in $L^{1}([0,1], r d r)$, then their Mellin convolution is defined by

$$
\left(f *_{M} g\right)(r)=\int_{r}^{1} f\left(\frac{r}{t}\right) g(t) \frac{d t}{t}, \quad 0 \leq r<1 .
$$

The Mellin convolution theorem states that

$$
\widehat{f *_{M} g}(s)=\widehat{f}(s) \widehat{g}(s)
$$

and that if $f$ and $g$ are bounded, then so is $f *_{M} g$.

It is important and helpful to know that the Mellin transform is uniquely determined by its value on an arithmetic sequence of integers. In fact we have the following classical theorem (see [8, p. 102]).

Theorem 2.4. Suppose that $f$ is a bounded analytic function on $\{z: \operatorname{Re} z>0\}$ which vanishes at the pairwise distinct points $z_{1}, z_{2}, \cdots$, where

i) $\inf \left\{\left|z_{n}\right|\right\}>0$ and

ii) $\sum_{n \geq 1} \operatorname{Re}\left(\frac{1}{z_{n}}\right)=\infty$.

Then $f$ vanishes identically on $\{z: \operatorname{Re} z>0\}$.

Remark 2.5. We shall use this theorem to show that if $\varphi \in L^{1}([0,1], r d r)$ and if there exists a sequence $\left(n_{k}\right)_{k \geq 0} \subset \mathbb{N}$ such that

$$
\widehat{\varphi}\left(n_{k}\right)=0 \text { and } \sum_{k \geq 0} \frac{1}{n_{k}}=\infty,
$$

then $\widehat{\varphi}(z)=0$ for all $z \in\{z: \operatorname{Re} z>2\}$ and so $\varphi=0$.

\section{Proofs of the theOREMS}

We start this section with the notion of radialization (see [11]). Letting $f$ be a bounded function on $D$, we define the "radialization" of $f$ by

$$
\operatorname{rad}(f)(z)=\frac{1}{2 \pi} \int_{0}^{2 \pi} f\left(e^{i t} z\right) d t .
$$

It is clear that a function $f$ is radial if and only if $\operatorname{rad}(f)=f$.

We are now ready to prove Theorem 1.1 .

Proof of Theorem 1.1. If $f$ is a quasihomogeneous function of degree $p$, then (a) is a direct consequence of Remark 2.2.

Conversely, suppose (a) is true. Then for any $k \in \mathbb{N}$,

$$
\begin{aligned}
T_{\bar{f}}\left(\bar{w}^{k}\right)(z) & =\left\langle\bar{f} \bar{w}^{k}, K_{z}+\overline{K_{z}}-1\right\rangle=\overline{\left\langle f w^{k}, K_{z}+\overline{K_{z}}-1\right\rangle}=\overline{T_{f}\left(w^{k}\right)(z)} \\
& =\overline{\lambda_{k}} r^{|k+p|} e^{-i(k+p) \theta} .
\end{aligned}
$$


Thus, if $p \geq 0$,

$$
\begin{aligned}
T_{\bar{z}^{p} f}\left(z^{k}\right) & =\left\langle\bar{w}^{p} f w^{k}, K_{z}+\overline{K_{z}}-1\right\rangle=\left\langle f w^{k}, w^{p} K_{z}\right\rangle+\left\langle\bar{w}^{p}, \bar{f} \bar{w}^{k}\left(\overline{K_{z}}-1\right)\right\rangle \\
& =\sum_{j=0}^{\infty}(j+1) z^{j}\left\langle T_{f}\left(w^{k}\right), w^{j+p}\right\rangle+\sum_{j=1}^{\infty}(j+1) \bar{z}^{j}\left\langle\bar{w}^{p}, T_{\bar{f}}\left(\bar{w}^{j+k}\right)\right\rangle \\
& =\sum_{j=0}^{\infty} \lambda_{k}(j+1) z^{j}\left\langle w^{k+p}, w^{j+p}\right\rangle+\sum_{j=1}^{\infty} \lambda_{j+k}(j+1) \bar{z}^{j}\left\langle\bar{w}^{p}, \bar{w}^{j+k+p}\right\rangle \\
& =\frac{\lambda_{k}(k+1)}{k+p+1} z^{k},
\end{aligned}
$$

and if $p<0$,

$$
\begin{aligned}
T_{z^{-p} f}\left(z^{k}\right) & =\left\langle f w^{k-p}, K_{z}+\overline{K_{z}}-1\right\rangle=\left\langle T_{f}\left(w^{k-p}\right), K_{z}+\overline{K_{z}}-1\right\rangle \\
& =\left\langle\lambda_{k-p} w^{k}, K_{z}+\overline{K_{z}}-1\right\rangle \\
& =\lambda_{k-p} z^{k} .
\end{aligned}
$$

In particular, if we denote $\varphi=r^{|p|} e^{-i p \theta} f$, then it follows that

$$
T_{\varphi}\left(z^{k}\right)=\delta_{k} z^{k}
$$

for some constant $\delta_{k} \in \mathbb{C}$. In the following we will prove that $\varphi$ is radial, which implies that $f$ is a quasihomogeneous function of degree $p$.

Now, writing out the integrals and changing the order of integration, we see that for each $m \in \mathbb{N}$,

$$
\begin{aligned}
\left\langle T_{\operatorname{rad}(\varphi)} z^{k}, z^{m}\right\rangle & =\frac{1}{2 \pi}\left(\int_{0}^{2 \pi} e^{i(m-k) t} d t\right)\left\langle T_{\varphi} z^{k}, z^{m}\right\rangle \\
& = \begin{cases}\left\langle T_{\varphi} z^{k}, z^{m}\right\rangle & \text { if } k=m \\
0 & \text { if } k \neq m .\end{cases}
\end{aligned}
$$

On the other hand,

$$
\left\langle T_{\operatorname{rad}(\varphi)} z^{k}, \bar{z}^{m}\right\rangle=\frac{1}{2 \pi}\left(\int_{0}^{2 \pi} e^{i(k+m) t} d t\right)\left\langle T_{\varphi} z^{k}, \bar{z}^{m}\right\rangle=0, \quad \forall m \in \mathbb{Z}_{+} .
$$

Then by (3.1), we obtain

$$
\left\langle T_{\operatorname{rad}(\varphi)} z^{k}, z^{m}\right\rangle=\left\langle T_{\varphi} z^{k}, z^{m}\right\rangle
$$

and

for all $m \in \mathbb{N}$. Thus

$$
\left\langle T_{\operatorname{rad}(\varphi)} z^{k}, \bar{z}^{m}\right\rangle=\left\langle T_{\varphi} z^{k}, \bar{z}^{m}\right\rangle
$$

$$
T_{\operatorname{rad}(\varphi)} z^{k}=T_{\varphi} z^{k}
$$

Moreover, by (3.1) and Lemma 2.3, we have

$$
T_{\varphi} \bar{z}^{k}=\delta_{k} \bar{z}^{k}, \quad \forall k \in \mathbb{N} .
$$

Then by a similar discussion, we can get

$$
T_{\operatorname{rad}(\varphi)} \bar{z}^{k}=T_{\varphi} \bar{z}^{k}
$$

From (3.2) and (3.3) we deduce that $T_{\operatorname{rad}(\varphi)}=T_{\varphi}$, which implies $\varphi(z)=\operatorname{rad}(\varphi)(z)$. Hence $\varphi$ is radial. This completes the proof.

Next we discuss the proof of Theorem 1.2 , 
Proof of Theorem 1.2. Suppose $f_{1}=e^{i k_{1} \theta} \varphi_{1}(r)$ and $f_{2}=e^{i k_{2} \theta} \varphi_{2}(r)$. Then for each $k \in \mathbb{N}$, it follows from Remark 2.2 that

$$
\begin{aligned}
T_{f_{1}} T_{f_{2}}\left(r^{k} e^{i k \theta}\right) & =T_{e^{i k_{1} \theta} \varphi_{1}} T_{e^{i k_{2} \theta} \varphi_{2}}\left(r^{k} e^{i k \theta}\right) \\
& =\lambda_{k_{2}, k} T_{e^{i k_{1} \theta} \varphi_{1}}\left(r^{\left|k+k_{2}\right|} e^{i\left(k+k_{2}\right) \theta}\right) \\
& =\lambda_{k_{1}, k+k_{2}} \lambda_{k_{2}, k} r^{\left|k+k_{1}+k_{2}\right|} e^{i\left(k+k_{1}+k_{2}\right) \theta}
\end{aligned}
$$

for some constants $\lambda_{k_{2}, k}$ and $\lambda_{k_{1}, k+k_{2}}$. Hence, if $T_{f_{1}} T_{f_{2}}=T_{h}$, then by Theorem 1.1, $h$ is a quasihomogeneous function of degree $k_{1}+k_{2}$. This completes the proof.

The following corollary, which characterizes the idempotent Toeplitz operators, is an immediate consequence of Theorem 1.2 .

Corollary 3.1. If $f$ is a quasihomogeneous function of degree different from 0 and if $T_{f}^{2}=T_{f}$, then $f=0$.

We now turn to the proof of Theorem 1.3 .

Proof of Theorem 1.3. Assume $T_{e^{i k_{1} \theta} r l_{1}} T_{e^{i k_{2} \theta} r_{2} l_{2}}$ is a Toeplitz operator. By Theorem 1.2, this operator is of the form $T_{e^{i\left(k_{1}+k_{2}\right) \theta} \psi(r)}$. Then for any $k \in \mathbb{N}$ such that $k+k_{2} \geq 0$ and $k+k_{1}+k_{2} \geq 0$, the equality $T_{e^{i k_{1} \theta} r^{l_{1}}} T_{e^{i k_{2} \theta} r^{2}}\left(z^{k}\right)=T_{e^{i\left(k_{1}+k_{2}\right) \theta} \psi}\left(z^{k}\right)$ together with Lemma 2.1 gives

$$
\widehat{r^{l_{1}}}\left(2 k+2 k_{2}+k_{1}+2\right) \widehat{r^{l_{2}}}\left(2 k+k_{2}+2\right)=\frac{1}{2 k+2 k_{2}+2} \widehat{\psi}\left(2 k+k_{2}+k_{1}+2\right) .
$$

Denote

$$
k^{\prime}=\min \left\{0, k_{1}, k_{2}, k_{1}+k_{2}\right\}
$$

Then by (2.1) and (3.4), we have

$\left(r^{l_{1}+k_{1}+k_{2}-k^{\prime}} *_{M} r^{l_{2}-k^{\prime}}\right)^{\wedge}\left(2 k+k_{2}+k^{\prime}+2\right)=\left(r^{k_{2}-k^{\prime}} *_{M} r^{k_{1}-k^{\prime}} \psi\right)^{\wedge}\left(2 k+k_{2}+k^{\prime}+2\right)$.

Next, by Remark 2.5. the above equation yields that

$$
r^{l_{1}+k_{1}+k_{2}-k^{\prime}} *_{M} r^{l_{2}-k^{\prime}}=r^{k_{2}-k^{\prime}} *_{M}\left(r^{k_{1}-k^{\prime}} \psi\right) .
$$

The above equation is equivalent to

$$
\int_{r}^{1} \psi(t) t^{k_{1}-k_{2}-1} d t=r^{l_{1}+k_{1}} \int_{r}^{1} t^{l_{2}-l_{1}-k_{1}-k_{2}-1} d t
$$

from which we can get

$$
\psi(r)= \begin{cases}\frac{l_{1}+k_{1}}{l_{1}+k_{-}-l_{2}+k_{2}} r^{l_{1}+k_{2}}-\frac{l_{2}-k_{2}}{l_{1}+k_{1}-l_{2}+k_{2}} r^{l_{2}-k_{1}} & \text { if } l_{1}+k_{1} \neq l_{2}-k_{2} \\ r^{l_{1}+k_{2}}\left(1+\left(l_{1}+k_{1}\right) \log r\right) & \text { if } l_{1}+k_{1}=l_{2}-k_{2} .\end{cases}
$$

Similarly, it follows from $T_{e^{i k_{1} \theta} r_{1} l_{1}} T_{e^{i k_{2} \theta} r_{2} l_{2}}\left(\bar{z}^{k}\right)=T_{e^{i\left(k_{1}+k_{2}\right) \theta} \psi}\left(\bar{z}^{k}\right)$ that

$$
r^{l_{1}-k^{\prime}} *_{M} r^{l_{2}+k_{1}+k_{2}-k^{\prime}}=r^{k_{1}-k^{\prime}} *_{M}\left(r^{k_{2}-k^{\prime}} \psi\right),
$$

from which we can get

$$
\psi(r)= \begin{cases}\frac{l_{1}-k_{1}}{l_{1}-k_{1}-l_{2}-k_{2}} r^{l_{1}-k_{2}}-\frac{l_{2}+k_{2}}{l_{1}-k_{1}-l_{2}-k_{2}} r^{l_{2}+k_{1}} & \text { if } l_{1}-k_{1} \neq l_{2}+k_{2} \\ r^{l_{1}-k_{2}}\left(1+\left(l_{1}-k_{1}\right) \log r\right) & \text { if } l_{1}-k_{1}=l_{2}+k_{2} .\end{cases}
$$

Since $l_{1}, l_{2}>0$, one can easily see that (3.5) and (3.6) are the same functions if and only if one of the following holds:

(1) $k_{1}=k_{2}=0$

(2) $l_{1}=l_{2}$ and $k_{1}=k_{2} \neq 0$, 
(3) $k_{1}=l_{1}$ and $k_{2}=l_{2}$,

(4) $k_{1}=-l_{1}$ and $k_{2}=-l_{2}$.

To show that only condition (1) holds, we next prove that conditions (2), (3) and (4) are all impossible to satisfy.

First, assume $l_{1}=l_{2}$ and $k_{1}=k_{2} \neq 0$. Without loss of generality, we can also assume that $k_{1}>0$ and $k_{2}>0$, for otherwise we could take the adjoints. Then it follows from (3.5) that

$$
\psi(r)=\frac{l_{1}+k_{1}}{2 k_{1}} r^{l_{1}+k_{1}}-\frac{l_{1}-k_{1}}{2 k_{1}} r^{l_{1}-k_{1}} .
$$

Now, consider the equality $T_{e^{i k_{1} \theta} r_{1} l_{1}} T_{e^{i k_{1} \theta} r^{l_{1}}}\left(\bar{z}^{k_{1}}\right)=T_{e^{2 i k_{1} \theta} \psi}\left(\bar{z}^{k_{1}}\right)$. Then we get

$$
\frac{4\left(k_{1}+1\right)}{\left(l_{1}+k_{1}+2\right)^{2}}=\frac{\left(l_{1}+k_{1}\right)\left(k_{1}+1\right)}{k_{1}\left(l_{1}+3 k_{1}+2\right)}-\frac{\left(l_{1}-k_{1}\right)\left(k_{1}+1\right)}{k_{1}\left(l_{1}+k_{1}+2\right)},
$$

and hence

$$
k_{1}\left(k_{1}+l_{1}\right)=0
$$

which is a contradiction since $k_{1}>0$ and $l_{1}>0$.

Next, assume $k_{1}=l_{1}$ and $k_{2}=l_{2}$. Then $\psi(r)=r^{l_{1}+l_{2}}$. So the equality $T_{z^{l_{1}}} T_{z^{l_{2}}}\left(\bar{z}^{l_{2}}\right)=T_{z^{l_{1}+l_{2}}}\left(\bar{z}^{l_{2}}\right)$ gives that

$$
\frac{2}{2 l_{2}+2}=\frac{2 l_{1}+2}{2 l_{1}+2 l_{2}+2},
$$

and hence

$$
l_{1} l_{2}=0
$$

which is a contradiction since $l_{1}>0$ and $l_{2}>0$.

Finally, assume $k_{1}=-l_{1}$ and $k_{2}=-l_{2}$. Similarly, it follows that $\psi(r)=r^{l_{1}+l_{2}}$. Then the equality $T_{\bar{z}^{l_{1}}} T_{\bar{z}^{l_{2}}}\left(z^{l_{2}}\right)=T_{\bar{z}^{l_{1}+l_{2}}}\left(z^{l_{2}}\right)$ gives that

$$
l_{1} l_{2}=0
$$

also a contradiction.

Consequently, if $T_{e^{i k_{1} \theta} r_{1} l_{1}} T_{e^{i k_{2} \theta} r_{2} l_{2}}$ is a Toeplitz operator, then $k_{1}=k_{2}=0$.

Conversely, the Toeplitz operator $T_{\psi}$, whose symbol $\psi$ given by

$$
\psi(r)= \begin{cases}\frac{l_{1}}{l_{1}-l_{2}} r^{l_{1}}-\frac{l_{2}}{l_{1}-l_{2}} r^{l_{2}} & \text { if } l_{1} \neq l_{2} \\ r^{l_{1}}\left(1+l_{1} \log r\right) & \text { if } l_{1}=l_{2}\end{cases}
$$

is obtained by taking $k_{1}=k_{2}=0$ in (3.5) and (3.6), takes the same values on $z^{k}$ and $\bar{z}^{k}$ for all $k \in \mathbb{N}$ as the product $T_{r^{l_{1}}} T_{r^{l_{2}}}$. This completes the proof.

\section{ACKNOWLEDGMENT}

We thank the referee for carefully reading the paper and providing corrections and suggestions for improvements.

\section{REFERENCES}

1. P. Ahern and Ž. Čučković, A theorem of Brown-Halmos type for Bergman space Toeplitz operators, J. Funct. Anal. 187 (2001), 200-210. MR.1867348 (2002h:47040)

2. A. Brown and P.R. Halmos, Algebraic properties of Toeplitz operators, J. Reine Angew. Math. 213 (1964), 89-102. MR0160136 (28:3350)

3. B.R. Choe and Y.J. Lee, Commuting Toeplitz operators on the harmonic Bergman spaces, Michigan Math. J. 46 (1999), 163-174. MR1682896(2000a:47054) 
4. Ž. Čučković and N.V. Rao, Mellin transform, monomial symbols, and commuting Toeplitz operators, J. Funct. Anal. 154 (1998), 195-214. MR.1616532 (99f:47033)

5. X.T. Dong and Z.H. Zhou, Algebraic properties of Toeplitz operators with separately quasihomogeneous symbols on the Bergman space of the unit ball, J. Operator Theory, to appear.

6. I. Louhichi, E. Strouse and L. Zakariasy, Products of Toeplitz operators on the Bergman space, Integral Equations Operator Theory 54 (2006), 525-539. MR2222982 (2007a:47033)

7. I. Louhichi and L. Zakariasy, On Toeplitz operators with quasihomogeneous symbols, Arch. Math. 85 (2005), 248-257. MR 2172383 (2006e:47061)

8. R. Remmert, Classical Topics in Complex Function Theory. Graduate Texts in Methematics, 172, Springer, New York, 1998. MR1483074 (98g:30002)

9. L. Zakariasy, The rank of Hankel operators on harmonic Bergman spaces, Proc. Amer. Math. Soc. 131 (2003), 1177-1180. MR1948109 (2003k:47038)

10. Z.H. Zhou and X.T. Dong, Algebraic properties of Toeplitz operators with radial symbols on the Bergman space of the unit ball, Integral Equations Operator Theory 64 (2009), 137-154. MR 2501175

11. N. Zorboska, The Berezin transform and radial operators, Proc. Amer. Math. Soc. 131 (2003), 793-800. MR.1937440 (2003h:47064)

Department of Mathematics, Tianjin University, Tianjin 300072, People's Republic OF CHINA

E-mail address: dongxingtang@163.com

Department of Mathematics, Tianjin University, Tianjin 300072, People's Republic OF CHINA

E-mail address: zehuazhou2003@yahoo.com.cn 